\title{
Intelligent Approach to Decision Support on Drug Abuse
}

\author{
B. Bali \\ Department of Computer \\ Science, \\ Adamawa State University, \\ Mubi, Nigeria
}

\author{
B. Y. Baha \\ Department of Computer \\ Science, \\ Taraba State University, \\ Jalingo, Nigeria
}

\author{
N. Nathan \\ Department of Computer \\ Science, \\ Adamawa State University, \\ Mubi, Nigeria
}

\begin{abstract}
Drug abuse is one of the highest occurrences of diseases globally, with increased cases of mental health, physical health and death problems. Drug control counselling centres and drug law enforcement agencies have made several efforts by implementing drug prohibition policies, campaign against drug abuse and creating awareness on risks associated with drug abuse. Unfortunately, drug abuse has become a global phenomenon affecting almost every country today. This problem can be greatly minimized by developing an intelligent system that can give assistance for such common situation. In this work, hybrid intelligent system has been developed for diagnosing drug abuse patients and recommend suitable advice that helps the patients reduce drug abuse problems. PHP.Net and MSQL were used for the development of the system. The system was tested and evaluated by the clinicians. The results generated from the system based on patient data confirm that the system can represent the expert's thinking in a satisfactory manner.
\end{abstract}

\section{General Terms}

Activity diagrams, conceptual frame work, drug abuse, and hybrid system.

\section{Keywords}

Intelligent decision support system, case-based reasoning, rule-based reasoning, drug abuse and intervention.

\section{INTRODUCTION}

Computer-based methods are now getting more and more involved in improving the quality of medicine and health sciences. According to [1], artificial intelligence has become a well-known topic of research and many systems have been developed in various domains to assist human for different kind of problems. The popular approach in artificial intelligence involves integrating components to offer overall advantages to systems. These systems are generally used in hospitals and clinics. Integrating intelligent systems have the potential to enhance the quality and efficiency of treatment [2]

Health is one of the most important aspects of human activities. The involvement of people in drug abuse is one of the major threats to health and the society at large [3] and [4]. Although drug control counselling centres, world health organization and drug law enforcement agencies among others have made several efforts by implementing drugprohibition policies, campaign against drug abuse and creating awareness on risks associated with drug abuse, drug abuse still persists, affecting almost every country today. [5] estimated global average of one in six people who suffer from drug-use disorders receive treatment each year, it is clear that the accessibility and availability of services for such conditions are limited in most countries, approximately 1 in 18 in Africa, compared with 1 in 5 in Western and Central Europe. In this aspect, the care of the drug abusers is very vital and their health needs given attention and support. The [6] listed the six types of psychoactive substances that can lead to drug abuse as follows: Inhalants, Narcotics, Stimulants, Depressants, Hallucinogens and Cannabis. Many researchers have carried out studies on the need for an intelligent system for decision support in investigating and recognizing diseases, their risks of infections, and possible solutions. However, Intelligent Decision Support Systems are yet to be implemented for drug abuse problems. This raised the need to develop a hybrid intelligent system that could diagnose and advice drug abusers. The aim of this research is to develop hybrid intelligent system for effective diagnosis and advice of drug abuse patients. The objectives are: to examine the feasibility of an intelligent approach in a drug abuse situation, to study signs and symptoms, threat effects and appropriate advice on drug abuse problems, to determine and justify the intelligent system model that fits in the field of drug abuse to support clinical workforce, and to develop a hybrid case-based and rule-based system capable of lessening drug abuse problems, This research work is significant in that it would help: to provide a hybrid case-based and rule-based system, to provide a suitable and improved way of diagnosing drug abusers, and to provide the knowledge available to young clinicians, and to provide fast and precise clinical advice that helps the drug abusers to reduce drug abuse attitude.

This paper is organized as into six (6) Sections. Section 1 presents brief background to study. Section 2, is concerned with a brief overview of several intelligent systems. In Section 3 , the proposed hybrid system is presented. Section 4 is concerned with the methodology. The details of the results are given in section 5. Finally, section 6 presents the conclusion of the work.

\section{LITERATURE REVIEW}

[7] constructed a knowledge-based hybrid expert system for diagnosis of neurologic disorders by encoding rules of the neurology domain and by developing a framework to learn from the cases of the patients. The constructed system exploited computer as an intelligent and logical device. The system is an attempt to improve the effectiveness of diagnosis in terms of accuracy, timeliness and quality. The diagnoses made by the system are similar to those made by a human expert which is in line with the intention of this work. [8] developed a medical diagnostic system applied to the acute bacterial meningitis, using the case based reasoning methodology. The research was focused on the implementation of the adaptation stage, from the integration of case based reasoning and rule based expert systems. [9] proposed decision support system for universal healthcare system with the use of decision support system. The decision support system was said to be capable of making decisions based on the diagnosis of estimated health situation in a comprehensive set of recommendations. [10] designed an 
expert system for diagnosis of blood disorder in human being. Forward chain and backward-chain approach are used to analyze the blood disorder which gives flexibility to the doctors on knowing more about a disease or diagnosing a patient through the system. The result of their analysis shows that the system can be used to capture diverse blood disorders. [11] stressed a compelling need for clinical decision support systems in electronic health records because it is indisputably the only way for clinicians of all types to take full advantage of the knowledge available to them. [12] stated that casebased reasoning has blown a fresh wind and a well justified degree of optimism into artificial intelligence in general, and knowledge based decision support systems in particular.

[1] presented a rule based expert system for diagnosing viral infections. The rule-based system was developed by converting the domain expert's knowledge into pattern-action format. They said the system was tested and the accuracy of the system was $90 \%$ achieved without code optimization.

[14] explained the two broad kinds of inference engines used in rule based expert systems. Forward chaining: is a data driven reasoning that involves receiving a problem description from the working memory as a set of conditions and tries to derive conclusions as a solution. It checks the condition part of the rule in the working memory to determine whether it is true or false. If the condition is true, then the action part of the rule is also true. Rule based reasoning technique uses conflict resolution strategy to select one rule at a time from the set. The selected rule is then applied to derive a conclusion about the problem. Backward chaining: starts by receiving the problem description as set of conclusions and working backward to find the premises or causes of the conclusions. Backward chaining is goal-driven reasoning that is very good when all the outcomes are known and the number of possible outcome is not large. Thus it is used when the solution is known while forward chaining is used when the absolute solution is not known. It searches the rules whose conclusion matches with part or all conclusions in the working memory. The selected rule is used to derive the premises that led to the given conclusion. According to [15], the challenge in this era where nearly all information are stored in digital format is how we can integrate, secure, and utilize amount of data in clinical decision support system to improve clinical outcomes. According to [16], rule-based reasoning (RBR) and casebased reasoning (CBR) are two complementary alternatives for building knowledge-based "intelligent" decision-support systems. [2] presented a system based on combination of case and rule-based reasoning for diagnosis and therapy of chronic obstructive pulmonary disease which is a very critical disease. Preliminary experimentations show that the expert system models well, the adaptation task and gives satisfactory answers to the user. The combination of CBR and RBR was made in order to gather their powers within the same system. [17] focused on combinations of case-based reasoning with rule based reasoning, and model based reasoning. Furthermore, they examined the strengths and weaknesses of various reasoning models, case-based reasoning, rule-based reasoning and model-based reasoning, and discussed how they can be combined to form a more robust and better-performing hybrid. However, they viewed combination of different problem solving and knowledge representation methods as a very active research area in Artificial Intelligence to provide more strength and less weakness. They also believed that complex problems are easier to solve with integrated approaches than individual approach. [18] proposed a weighted hybrid system that integrates multiple recommendation algorithms to improve performance of collaborative filtering $(\mathrm{CF})$ recommender system. The scheme combines two model-based methods RCF and CCF by utilizing the techniques: clustering RBR, and CBR. Experimental results reveal that the proposed system consistently outperforms the individual recommendation algorithms in terms of prediction and classification accuracy. [19] described the four major functions of an intelligent system. These include performing administrative tasks such as documenting the patient records and referrals, managing the clinical difficulties and details, cost control by eliminating the redundant and unnecessary tests and finally providing the decision support. These views are in line with the researcher's intent.

\section{SYSTEM ARCHITECTURE}

The system is titled Hybrid Intelligent System, to diagnose and give clinical advice on drug abuse issues. This system was designed using the PHP.net Platform and MySQL Database.

The architecture describes how different classes and modules in the system interact. The hybrid reasoning system make the diagnosis much more accurate than when there is only one reasoning system. The rate of diagnosis increases as the CBR module takes care of cases which cannot be solved by the RBR module. There are different modules for arriving at a diagnosis and for suggesting an appropriate advice. Rules represent general knowledge of the domain, whereas cases represent the specific knowledge. Rule-based systems solve problems from scratch, while case-based systems use prestored situations to deal with similar new instances.

The interaction between the two reasoning methods can be done by sending messages. Figure 1 below represents the architecture of the system. The case based reasoning method receives the input case and provides it to the rule based reasoning method for further explanation. It does the same after it retrieves a case from the case base. The rule based reasoning method interferes between retrieving a case and case matching processes of the case based reasoning method. The case based component calls the rule based reasoning method with a message of the case to be explained. When the rule based reasoning method has finished its conversion, it calls the case based reasoning method with a message of the converted case.

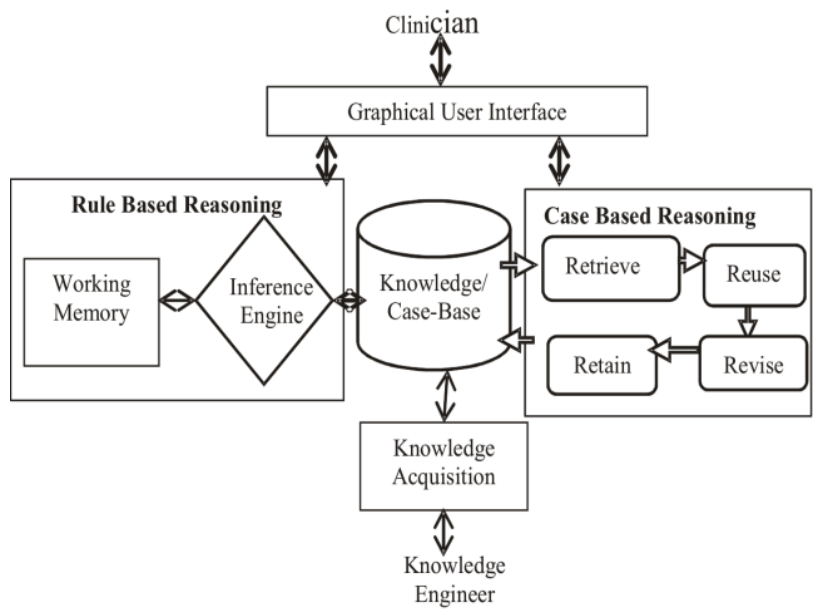

Figure 1: Architecture for hybrid intelligent system

Knowledge base contains a collection of facts and rules used to make decisions. This information presents knowledge gained from qualified experts in the field, in the form of facts 
and rules. Generally, rules consist of if-then statements, where a given set of conditions will lead to a specified set of results. Working memory contains information that is supplied by the end user. This information is used to evaluate antecedents in the knowledge base. A change in the knowledge base results in creation of new values, thus the working memory will update its old values. Case base contains new and previous cases for retrieving similar cases and as data storage. The main function of the case-based component is to perform case matching. User interface is an intermediary that allows communication between the user and the system. The purpose of the user interface is to ease the usage of system by users and administrators, by providing forms for taking input to the system such as symptoms or signs, and screen to present output to the user. Inference engine maneuvers knowledge from both bases for searching the most relevant cases for a new given case. It seeks information and relationship from the knowledge base and to provide answers, predictions and suggestions in the way a human expert provides. The inference engine receives a problem from the working memory and provides the reasoning result to the working memory. It determines which rules to apply to a given question, and in what order, by using information in the knowledge base. Forward chaining reference engine: a data driven reasoning involves receiving a problem description from the working memory as a set of conditions and tries to derive conclusions as a solution. It checks the condition part of the rule in the working memory to determine whether it is true or false. If the condition is true, then the action part of the rule is also true. This procedure continues until the solution is found. Rule based reasoning technique uses conflict resolution strategy to select one rule at a time from the set. The selected rule is then applied to derive a conclusion about the problem. The initial diagnostic process is done through firing of rules in the Rule-Based inference.

\subsection{Conceptual framework of the study}

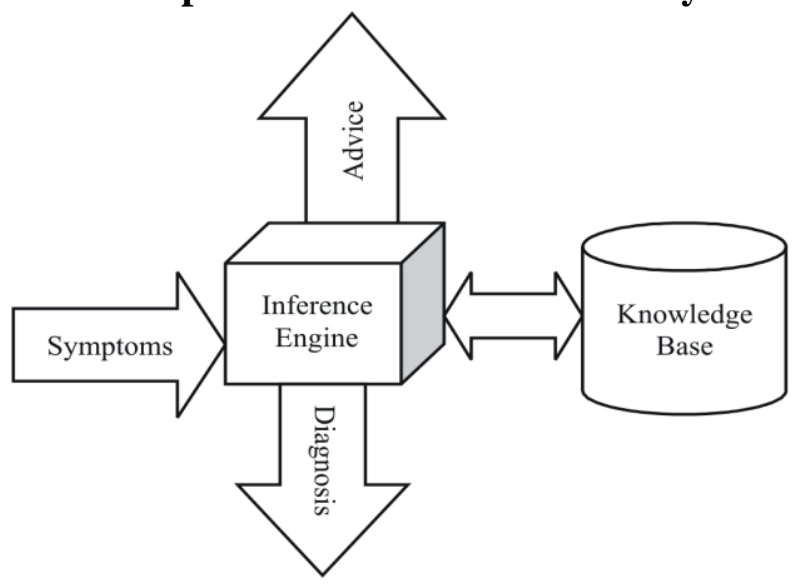

Figure 2: Working model of hybrid intelligent system

Figure 2 represents how the symptoms are provided, consultations were made with the knowledge base and advice was retrieved accordingly. The symptoms, effects and advice reside in the knowledge base in the form of rules. The inference engine is the component having the reasoning capabilities. The reasoning in the system is just like emulating the physician while interacting with a patient for diagnosis, by asking questions about signs and symptoms and give advice based on the provided symptoms.

\section{METHODOLOGY}

Activity Diagram: shows the activity in both the computational and organizational processes. It captures the dynamic behaviour of the system.

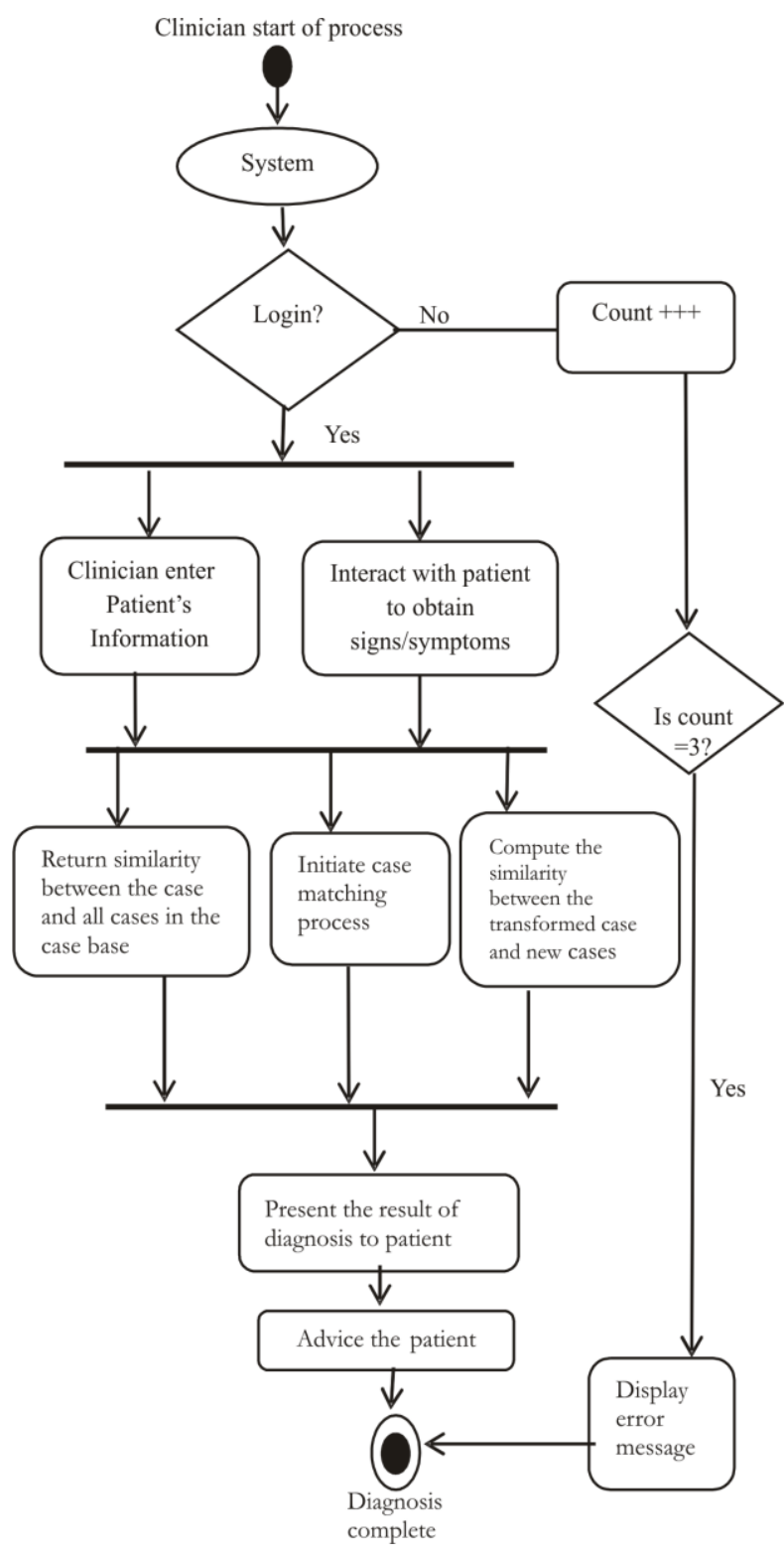

Figure 3: Activity diagram of the system.

Knowledge acquisition: Knowledge acquisition is the process of collecting data from knowledge source to computer code by interviewing experts in the domain. Protocolgeneration techniques which include a variety of types that range from interviews, to reporting techniques and observational techniques were used. The knowledge is acquired by consulting an expert in the medical field to find out how the problem is solved. This involves interaction with the domain expert to acquire relevant information such as signs, symptoms, effects, observation of diagnosis of a patient and suitable advice or solution. Related literatures to drug abuse effects were reviewed.

The possible signs and symptoms of drug abuse patients were the parameters for designing the system as shown in table 3.1 below. 
Table 3.1: Showing symptoms list

\begin{tabular}{|l|l|l|l|l|l|l|}
\hline No & Symptom & wt. & No & Symptom & wt. \\
\hline P01 & Agitation & 3 & & P15 & $\begin{array}{l}\text { Increased } \\
\text { pupil }\end{array}$ & 3 \\
\hline P02 & Anxiety & 4 & & P16 & Insomnia & 3 \\
\hline P03 & Bad Breath & 3 & & P17 & Lethargy & 3 \\
\hline P04 & Confusion & 3 & & P18 & Nausea & 1 \\
\hline P05 & Constipation & 3 & & P19 & Paranoia & 4 \\
\hline P06 & Constricted & 3 & & P20 & Shallow \\
pupils & & & 4 \\
\hline P07 & $\begin{array}{l}\text { Dilated pupils } \\
\text { P08 }\end{array}$ & 2 & P21 & $\begin{array}{l}\text { Slowed } \\
\text { breathing }\end{array}$ & 3 \\
\hline P09 & Drowsiness & 2 & & P23 & Sweating & 4 \\
\hline P10 & Euphoria & 1 & & P24 & Talkativeness & 4 \\
\hline P11 & Excitation & 4 & & P25 & Tremors & 3 \\
\hline P12 & Hallucinations & 3 & P26 & Vomiting & 3 \\
\hline P13 & $\begin{array}{l}\text { Impaired } \\
\text { judgement }\end{array}$ & 4 & P27 & Weight loss & 3 \\
\hline P14 & Incoordination & 3 & & P28 & Dry lips & 4 \\
\hline
\end{tabular}

\section{SYSTEM TESTING}

\subsection{Home Page}

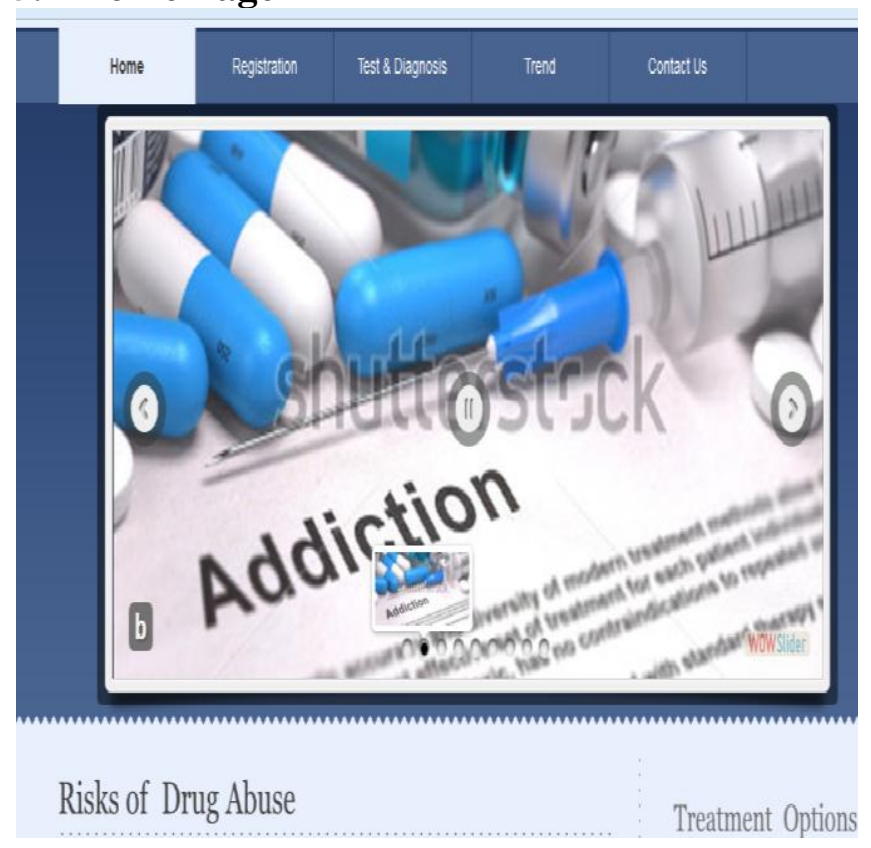

Figure 4: Home page

\subsection{Diagnosis Window}

This window displays the list of possible symptoms associated with the drug abuse effects. After the submit button is clicked, the consultation result is added to the patient medical history as this can be useful for making important future medical decision on the patient health.

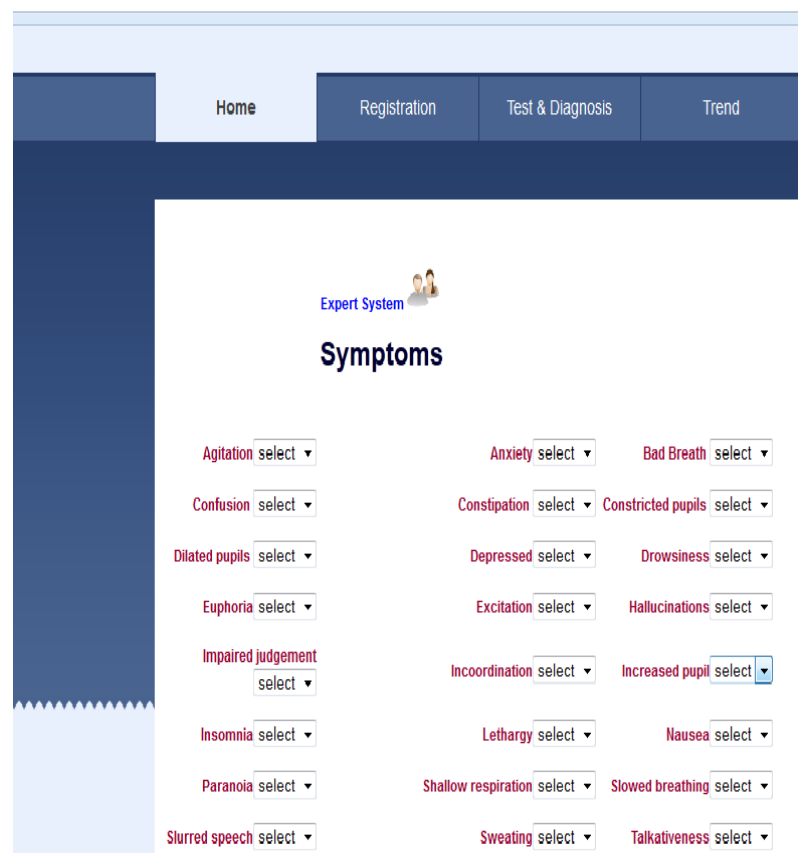

Figure 5: Diagnosis window

\subsection{Result Discussion}

\section{Drug̣g Addictions Expert System}

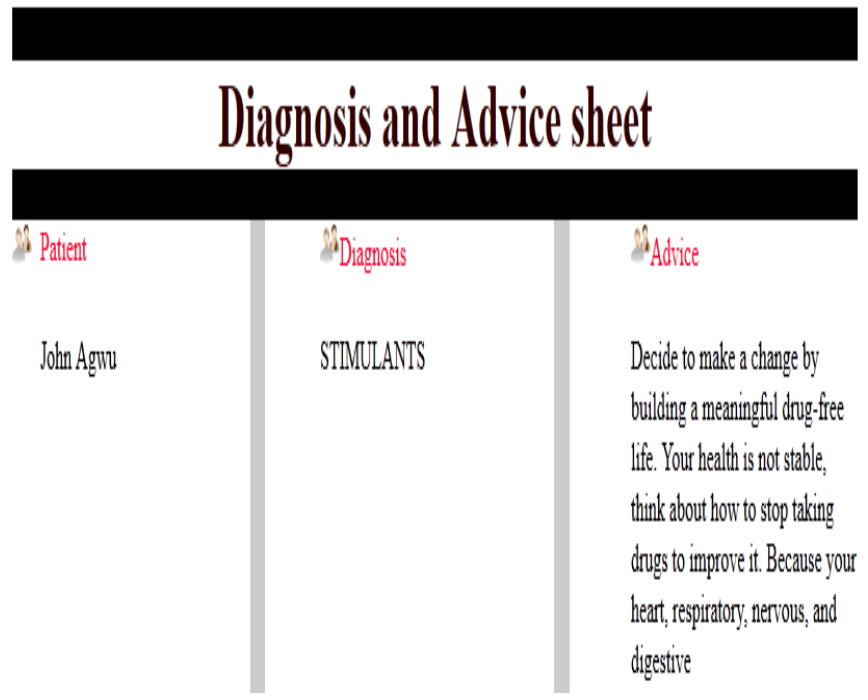

Figure 6: Test result

Our work studied and designed how a rule based reasoning component can be developed and integrated with the case based reasoning. The system developed demonstrated the hybrid power of the rule based reasoning method on case transformation to support the case based reasoning on drug abuse patient in clinical domain. The hybrid system was implemented based on the design discussed and proposed in Figure 1. The results obtained represents the expert's thinking in a satisfactory manner in handling patients' diagnosis and 
suggestion for a healthy living. Furthermore, it provides a very fast and precise diagnosis and minimizes the amount of time the medical personnel spend in attending to patients suffering from drug effect. The results have shown that the system provides a clinically relevant tool. The developed system for diagnosis of drug abuse effect is a medical expert system. Using this system user will be conversant with the symptoms and causes of drug effects and give suggestion for a healthy living. The intelligent system designed run on the PHP.net and MySQL as backend.

\section{CONCLUSIONS}

This work discussed the development of a hybrid intelligent system for diagnosis and advising drug abuse patients. The focus was set on adapting existing case-based and rule-based reasoning and their integration and presented detailed analysis and research reports on relevant research reports on drug abuse issue. These findings are important as the study demonstrate a new system that would assist clinicians in diagnosing drug abuse patients. The intelligent system designed run on the PHP.net and MySQL as backend. The constructed system attempts to improve the effectiveness of diagnosis and to provide a clinically relevant intelligent tool. However, few properties of this model remain to be investigated.

\section{Further study}

As this study is a pioneering work, there is a need for more research on the intelligent approach to drug abuse in the future. Future research would consider:

i. development of web-based intelligent approach to decision support on drug abuse,

ii. design of web based intelligent system for specific drug abuse effect could also be carried out in later works, and

iii. implementation using different technologies such as artificial neural network, fuzzy logic and java technology.

\section{ACKNOWLEDGMENTS}

I would like to acknowledge all the resources available at the Adamawa State University, Mubi and all authors of the journal articles used to complete this research work.

\section{REFERENCES}

[1] Patel, M., Patel, A., \& Virparia, P. (2013). Rule based expert system for viral infection diagnosis. International journal of advanced research in computer science and software engineering, 3 (5), 591-591.

[2] Guessoum, S., Laskri, T. M., djellali, H., \& Khadir T. M. (2012). Combining case and rule based reasoning for the diagnosis and therapy of chronic obstructive pulmonary disease. International journal of hybrid information technology, 5 (3), 145-160.

[3] Omadjohwoefe, S. O. (2010). Drug prohibition and the problem of conformity in Nigeria. $J$ psychology, 1 (2), 91-97.

[4] Mental Health First Aid Australia (2013). Helping someone with drug use problems. Mental health first aid guidelines. Melbourne. 1-4.

[5] UNODC (2015). World drug report 2015. United nations office on drugs and crime, United Nations, New York. 19
[6] NIDA (2010). Drugs, brains and behavior: The science of addiction, National institute of health $U . S$. department of health and human resources, 3-14.

[7] Mathew, S., Mathew, S., Hamed, H. M., \& Qadri, I. (2015). A web based decision support system driven for the neurological disorders. International journal of engineering research and general science, 3 (4), 177192.

[8] Cabrera, M. M., \& Edye, O. E. (2010). Integration of rule based expert systems and case based reasoning in an acute bacterial meningitis clinical decision support system. International journal of computer science and information security, 7 (2), 112-118.

[9] Conejar, J. R., \& Kim, K. H. (2014). A medical decision support system (DSS) for ubiquitous healthcare diagnosis system. International journal of software engineering and its applications. 8 (10), 237-244.

[10] Ayangbekun, O. J., Olatunde, A. I., \& Bankole, O.F. (2014). An expert system for diagnosis of blood disorder. International journal of computer applications, 100(7) $0975-8887$.

[11] Morrison, D. (2012). Clinical decision support systems (CDSS) within behavioral healthcare settings. Netsmart, 1-9.

[12] Voskoglou, G. M. \& Salem, M. A. (2014). Analogybased and case-based reasoning: two sides of the same coin. International journal of applications of fuzzy sets and artificial intelligence, 4, 5-51.

[13] Hole, R. K., and Gulhane, S. V. (2014). Rule-based expert system for the diagnosis of memory loss diseases. International journal of innovative science, engineering and technology, 1 (3), 80-83.

[14] Castaneda, C., Nalley, K., Mannion, C., Bhattacharyya, P., Blake, P., Pecora, A., Goy, A., \& Suh, S. K. (2015). Clinical decision support systems for improving diagnostic accuracy and achieving precision medicine. Journal of clinical bioinformatics, 5(4), 1-16.

[15] Berka, P. (2011). A compositional approach to rulebased and case-based reasoning. Advances in artificial intelligence. 1-16.

[16] Khandelwal, K. \& Sharma, P.D. (2013). Hybrid reasoning model for strengthening the problem solving capability of Expert Systems. International journal of advanced computer science and applications, 4 (10), 8894.

[17] Tyagi, S. and Bharadwaj, K. K. (2012). A Hybrid Recommender System Using Rule-Based and CaseBased Reasoning, International journal of information and electronics engineering, 2 (4), 586-590.

[18] Kong G, Xu D. L, Yang J. B. (2008) Clinical decision support systems a review on knowledge representation and inference under uncertainties, International Journal of Computational Intelligence Systems, Vol.1, No. 2, 159167. 